\title{
Opportunities for RTL and Gate Level Simulation using GPUs (Invited Talk)
}

\author{
Yanqing Zhang, Haoxing Ren, Brucek Khailany \\ NVIDIA Corporation \\ \{yanqingz,haoxingr,bkhailany\}@nvidia.com
}

\begin{abstract}
This paper summarizes the opportunities in accelerating simulation on parallel processing hardware platforms such as GPUs. First, we give a summary of prior art. Then, we propose the idea that coding frameworks usually used for popular machine learning (ML) topics, such as PyTorch/DGL.ai, can also be used for exploring simulation purposes. We demo a crude oblivious two-value cycle gate-level simulator using the higher level ML framework APIs that exhibits $>20 \mathrm{X}$ speedup, despite its simplistic construction. Next, we summarize recent advances in GPU features that may provide additional opportunities to further state-of-the-art results. Finally, we conclude and touch upon some potential areas for furthering research into the topic of GPU accelerated simulation.
\end{abstract}

ACM Reference Format:

Yanqing Zhang, Haoxing Ren, Brucek Khailany . 2020. Opportunities for RTL and Gate Level Simulation using GPUs (Invited Talk). In IEEE/ACM International Conference on Computer-Aided Design (ICCAD '20), November 2-5, 2020, Virtual Event, USA. ACM, New York, NY, USA, 5 pages. https: //doi.org/10.1145/3400302.3415773

\section{Introduction}

Gate-level and RTL simulation play an important role in the design of integrated circuits. Simulation is ubiquitous in many design steps such as functional verification, fault testing, as well as performance and power validation. These design tasks require long simulation times due to growing chip sizes (more logic gates or processes to simulate) and functionality (more testbenches to simulate). Thus, there is a desire to accelerate simulation. Modern day parallel computing architectures, such as GPUs (graphics processing units), provide an opportunity for speedups through parallelization across cycles (stimuli) as well as the design (logic gates). GPUs also present challenges to simulation acceleration, such as code translation to be able to run on GPU hardware. Other challenges include overcoming obstacles to substantial speedup such as minimizing communication overhead between host/device (CPU/GPU), efficient scheduling, and optimizing memory access patterns [2-7].

Indeed, much extensive prior research has been conducted on the topic of gate level and RTL simulation speedup using GPUs with impressive results [2-6]. By utilizing GPUs for parallelization, they achieve up to $1000 \mathrm{X}$ order of magnitude speedups [2]. This is despite that simulation has been classified as a difficult program to parallelize $[4,8]$ due to the sparsity of graph (the simulated netlist

Permission to make digital or hard copies of all or part of this work for personal or classroom use is granted without fee provided that copies are not made or distributed for profit or commercial advantage and that copies bear this notice and the full citation on the first page. Copyrights for components of this work owned by others than the author(s) must be honored. Abstracting with credit is permitted. To copy otherwise, or republish, to post on servers or to redistribute to lists, requires prior specific permission and/or a fee. Request permissions from permissions@acm.org.

ICCAD '20, November 2-5, 2020, Virtual Event, USA

(c) 2020 Copyright held by the owner/author(s). Publication rights licensed to ACM. ACM ISBN 978-1-4503-8026-3/20/11...\$15.00

https://doi.org/10.1145/3400302.3415773

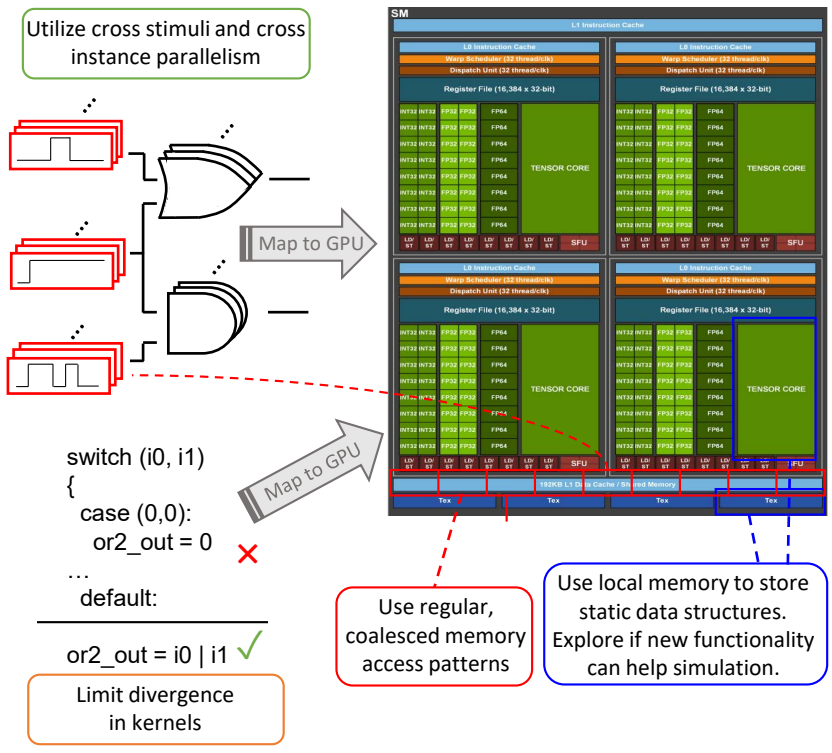

Figure 1: Depiction of key techniques commonly used for GPU acceleration. The techniques include: 1. Maximizing GPU utilization through parallelism across different input stimuli and instances in the same logic level. These aspects are easily parallelized since their simulation is independent of each other. 2. Limiting divergence in kernel code 3. Memory organization schemes for regular, coalesced access patterns. 4. Exploring methods that utilize the newest GPU and CUDA (or other programming API) features. Diagram of NVIDIA Ampere GPU SM taken from [1].

data is stored as a graph structure) algorithms, compared to other more amenable EDA tasks such as logic synthesis optimization, floorplanning, and routing.

Simulators fall into two main schools of thought: oblivious and event-driven. Oblivious simulation evaluates every gate or process at every cycle or timestamp, while event-driven simulation only performs evaluation on those gates and processes that have value changes on their inputs. Each type of simulator has its own merits and pitfalls, and create unique opportunities and challenges to GPU adaptation. Oblivious simulators have low control overhead and simplistic code, leading to easy mapping to GPUs. However, oblivious simulation can be very computationally inefficient when activity rate is low as it needlessly simulates gates/processes that are idle. In addition, it strains the need for memory space and bandwidth as every signal at every timestamp must be recorded. In contrast, event-driven simulators' computation is very efficient as it only simulates gates/processes that exhibit activity. However, they require a dynamic runtime scheduler, which leads to more complex code that is not as easy to map to GPUs. In addition, eventdriven simulators require book-keeping tasks regarding which and when gates/processes toggle, leading to increased communication 
overhead on the GPU and more irregular memory access patterns. In our survey of prior art, we find that event-driven simulation tends to garner larger speedup numbers [2,3]. Event-based simulation is also the predominant method used by commercial simulation tools.

While GPU accelerated simulation is a challenging topic, we propose two key opportunities that can help in pushing the stateof-the-art forward: the advent of machine learning (ML) coding frameworks (such as PyTorch [9] and/or DGL.ai [10]) which lower the barrier of entry in exploration of the topic, and newer GPU hardware, which have optimizations that directly boost GPU accelerated simulation prospects. These opportunities are discussed in detail in Sections 3 and 4, respectively.

\section{Background: A Summary of Prior Art}

Figure 1 summarizes some of the main ideas and techniques prior art has utilized to unleash GPU accelerated speedups. While we leave the detailed merits of each prior work to the respective citation itself, we next try to give a brief summary of each.

[2] proposes an event-driven simulator idea for a gate level simulator of logic gates where the primary inputs and pseudo-primary inputs (output pins from sequential cells such as flip-flops, register files, or SRAMs) are known. The simulation of each gate instance is assigned to a GPU thread. In this way, GPU parallelization across multiple different stimuli and logic instances in the same logic level is enabled, because simulation of those separate instances are independent of one another. They achieve $21 \mathrm{x}-1000 \mathrm{X}$ speedup. Divergences in code, which greatly slow down thread execution on the GPU, is kept to a minimum by having a minimalist simulation kernel, and synchronization happens only at the boundary of different logic levels of the levelized gate netlist. The authors spend a great deal of effort with a novel memory organization method to make memory access patterns regular, coalesced, and reusable (flushing stale intermediate simulation values). They also make use of on-GPU-device, fast static memory for static data structures such as graph data of the DUT netlist. Some critiques of the idea may be that though simulation is timing aware, no sequentials are simulated, and much memory-wrangling and calibration is needed to get optimal speedups.

[3] proposes a similar event-driven simulator. The authors focus on lowering communication overhead involved in event-driven simulation. They do so by assigning separate thread blocks to the 3 kernels needed for simulation: extract (sending output pin signal values outward), fetch (input pins receiving driver net value changes), and evaluate (actually performing the boolean logic function). They employ a unique memory paging mechanism to allow a wider range of signal activity toggle rates to be dynamically and efficiently stored. They also cleverly use fast static memory on the GPU (such as texture cache or constant memory) to store non-changing data structures (such as truth-tables and netlist graph structure data). Lastly, to further reduce host/device communication, they make use of a new (at the time) CUDA feature, asynchronous execution, to mask communication runtime. This perhaps implies that as further and newer features within GPUs and their programming APIs are released, more optimization opportunities may present themselves for accelerating simulation. Unfortunately, this method still sometimes struggles with speeding up low activity rate simulations (5-270X speedup).

In contrast to $[2,3],[4,5]$ take an oblivious approach to cycle based gate level simulation. They make use of heuristic clustering techniques to reduce the synchronization overhead of simulating logic level by level through partitioning the netlist into 'macro gates' spanning several logic stages. The authors take care not to disrupt the independent nature between different threads' execution, despite each thread now being responsible for simulating more than one gate instance. These methods also utilize the common practices of translating the netlist into a directed acyclic graph (DAG) to store on the GPU device, and doing clever memory organization to coalesce memory read patterns to retrieve signal values. They achieve a range of 5-45X speedup.

While most prior art focuses on the topic of boolean logic gate level simulation, the authors of $[6,7]$ observe that the idea of GPU accelerated simulation can be expanded to higher level languages as well, such as RTL [6] and SystemC [7] simulation.

In [6], the authors take the method proposed in [3] and expand it to RTL simulation. The framework uses every RTL process (e.g., always and initial blocks in Verilog) as the basic 'standard cell' building block instead of logic gates to build an RTL dependency graph and assign each 'RTL gate' to a different warp (a group of 32 threads) on the GPU. They purposefully lose one level of GPU mapping granularity (as opposed to mapping gate instances to thread granularity as described above) based on the observation that most RTL processes will not have similar code, so mixing processes within a warp will greatly slow thread execution down. In addition, they cleverly translate the code in each RTL process into CUDA [11] code that can be directly compiled with standard CUDA development tools. New (at the time) CUDA functionality aided in their idea: pointers to device functions quickened code loading times for the different RTL processes, and context switching greatly improved GPU utilization. They achieve 20-50X speedups while mentioning the communication overhead of writing results back into global memory became a main hindrance to bigger speedups. Of note is that this method focuses on scaling GPU accelerated simulation through 'gate' parallelism, as opposed to 'strong scaling' a single testbench/stimulus which may run into Amdahl's law bottlenecks. It shows that ideas such as utilizing cross instance and stimuli parallelism can be ubiquitous across different levels of simulation (and not restricted to gate level simulation).

\section{Opportunity: Machine Learning Frameworks}

The popularity of ML applications (which benefit from parallelization themselves) has led to many machine learning frameworks such as PyTorch [9], which use higher level languages such as Python to lower the barrier of entry of coding in parallel applications, thereby accelerating the process from research and code development to deployment and experimentation. For this reason, PyTorch is also widely used in the ML for EDA domain [12]. These frameworks have in turn led to many useful ML packages being developed, some of which we believe are especially useful for accelerated GPU simulation purposes. One such package is DGL.ai (Deep Graph Library), which is a package geared toward graph neural networks (GNN) [13] and graph learning. Because a netlist is a graph, there are many APIs in the package that conveniently provide functionality that simulation will need, such as netlist levelization and message/signal propagation methods. Also, the package itself has optimizations for graph analysis speed and memory storage requirements. As a demonstration, we exemplify how even the most simplistic programs can achieve simulation speedups by re-purposing PyTorch/DGL.ai for gate simulation. Namely, we build an oblivious, two-value cycle based (zero delay mode) simulator where the inputs and pseudo-inputs are given (much like the scenario in [2]).

Data Structure Setup: The main data structures, as depicted in Figure 2, in our demo simulator are: a 2D waveform tensor of size [gates/nodes, cycles] that stores the $0 / 1$ waveforms of each 


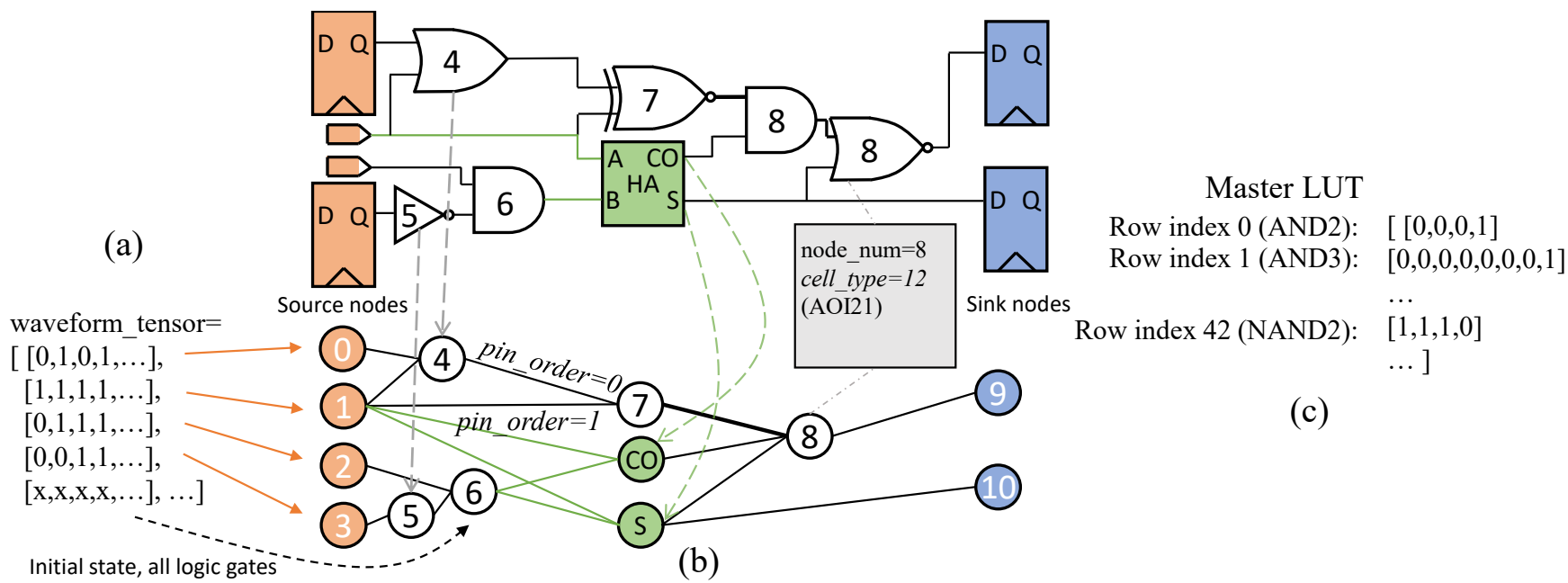

Figure 2: Depiction of data structure setup for demo simulator. (a) waveform-tensor. (b) graph object. (c) master lookup table.

gate/node (Figure 2(a)), a DGL.ai graph object that represents the netlist (Figure 2(b)), and a 2D tensor master LUT of size [cell types, evaluated value columns] that houses all the evaluated values for all input combinations across all boolean logic gate types (Figure 2(c)) The graph object is created by taking a verilog netlist and first translating it into a Python boolean sparse lil_matrix object, where each row/column entry corresponds to a gate in the netlist, or a node in the eventual graph (from here on, we use gate/node and net/edge interchangeably). A 'True' entry signifies an edge exists between nodes (Some nodes may be duplicated if the gate has multiple outputs, such as a full-adder cell). We 'cut' the graph based on pipeline stages by designating sequential output pins as source nodes and sequential input pins as sink nodes. From here, APIs in DGL.ai exist to transform the lil_matrix into a DGL.ai graph object. We also collect information regarding cell type and which cell pin each net is driving. This information is conveniently stored in the graph object by way of graph node and edge features. Figure 2(b) and Figure 4 exemplifies how this is done. Each node is given a cell_type node feature, which is an integer number representing the cell type (for example, 0 can represent AND2 logic, 1 can represent AND3 logic, etc). Each edge is given a pin_order edge feature, which is an integer representing which pin the net/edge is driving (for

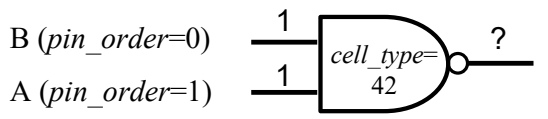

Every edge/pin has a pin order feature. If the input to the pin is a 1, then this pin contributes $2^{\wedge}$ (pin_order) rows to this cell's 'binary-ordered' truth-table (below). In this waveform, we have $2^{\wedge} 0+2^{\wedge} 1=3$, so we will look at row 3 of the truth-table for NAND2, the answer is $\mathrm{Y}=0$

\begin{tabular}{|c|c|c|c|c|}
\hline Row \# & A & B & $\mathbf{Y}$ & \multirow{5}{*}{$\begin{array}{l}\text { make Y a vector, insert into cell type } \\
(=42) \text { row of master LUT. The column } \\
\text { index of the Y vector corresponds to the } \\
\text { binary-ordered truth-table row index. }\end{array}$} \\
\hline 0 & 0 & 0 & 1 & \\
\hline 1 & 0 & 1 & 1 & \\
\hline 2 & 1 & 0 & 1 & \\
\hline 3 & 1 & 1 & 0 & \\
\hline
\end{tabular}

Figure 3: Mechanism behind construction of master LUT. example, 0 and 1 can represent the 2 AND inputs in an AOI21 gate, while 2 can represent the one OR input).

Figure 3 gives a depiction of how each row of the master LUT is created. The master LUT stores the evaluated values of all cell types. Each row's entry corresponds to the cell type matching the graph's cell_type node feature (so row 0 will store AND2 logic evaluation results, row 1 will store AND3 logic results, etc). Each column stores the result of logic evaluation of the cell type when the weighted sum (weighted based on the pin_order edge feature) of all the cell's input pins equal the column index. It will become apparent why we do this in the next section.

Finally, each row of the waveform tensor houses the resulting per cycle waveform of each gate. The row index matches the node index (node_num) for each gate in the graph. Sequential output pin (source node) rows will be pre-populated with the correct simulation waveforms at the start of simulation in our scenario (Figure 2(a)).

Execution Code. With the above data setup, the remaining tasks left to complete the simulator is a mechanism to load the signals to the correct nodes of a levelized graph, propagate the signals from one level to the next, perform a table look-up based on the received signal values to evaluate the boolean logic expression of each node, update the corresponding rows of the waveform tensor, and repeat all the above steps for each level of the graph. Figure 4 gives a summary of these tasks and their corresponding

Table 1: Summary of Simulation Benchmarks

\begin{tabular}{|c|c|c|c|}
\hline Design & Description & $\begin{array}{l}\text { Gate } \\
\text { Count }\end{array}$ & Stimulus \\
\hline qadd_pipe & 32-bit fixed point adder & 774 & $\begin{array}{l}\text { Random } \\
\text { stimulus }\end{array}$ \\
\hline qmult_pipe & 32-bit fixed point multiplier & 1410 & $\begin{array}{l}\text { Random } \\
\text { stimulus }\end{array}$ \\
\hline fadd & 32-bit floating point adder & 961 & $\begin{array}{l}\text { Random } \\
\text { stimulus }\end{array}$ \\
\hline fmult & 32-bit floating point multiplier & 2005 & $\begin{array}{l}\text { Random } \\
\text { stimulus }\end{array}$ \\
\hline NoCRouter & $\begin{array}{l}\text { Wormhole router with virtual } \\
\text { channels }\end{array}$ & 10,330 & $\begin{array}{c}\text { Specific } \\
\text { operation } \\
\text { mode tests }\end{array}$ \\
\hline $\begin{array}{l}\text { RISC-V } \\
\text { Core }\end{array}$ & $\begin{array}{l}\text { RISC-V Rocket Core } \\
\text { (SmallCore) }\end{array}$ & 56,243 & $\begin{array}{l}\text { open source } \\
\text { benchmarks }\end{array}$ \\
\hline
\end{tabular}


Table 2: GPU Accelerated Simulation Speedup Results

\begin{tabular}{cccccccc} 
Design & $\begin{array}{c}\text { Max \# of } \\
\text { Cycles } \\
\text { Batched }\end{array}$ & $\begin{array}{c}\text { Simulation } \\
\text { Time }(\mathbf{s})\end{array}$ & $\begin{array}{c}\text { "GPU } \\
\text { Throughput" } \\
\text { (gate * } \text { cycles/s) }\end{array}$ & $\begin{array}{c}\text { GPU Sim } \\
\text { Throughput } \\
\text { (cycles/s) }\end{array}$ & $\begin{array}{c}\text { Baseline } \\
\text { Throughput } \\
\text { (cycles/s) }\end{array}$ & $\begin{array}{c}\text { Speedup } \\
\text { (X) }\end{array}$ & $\begin{array}{c}\text { Toggle } \\
\text { rate }\end{array}$ \\
\hline $\begin{array}{c}\text { qadd_pipe } \\
\text { qmult_pipe }\end{array}$ & 400000 & 3.39 & $91,151,812$ & 117994.1 & 686.5 & 171.9 & 0.4515 \\
fadd & 240000 & 1.35 & $83,682,090$ & 59259.3 & 164.0 & 361.3 & 0.4789 \\
fmult & 80000 & 2.60 & $88,594,782$ & 92307.7 & 1252.6 & 73.7 & 0.0553 \\
NoCRouter & 21200 & 2.77 & $90,405,851$ & 45197.7 & 809.7 & 55.8 & 0.0552 \\
RISC-V & 5400 & 3.97 & $88,460,955$ & 8583.0 & 60.6 & 141.6 & 0.0418 \\
Core & & $76,428,612$ & 1360.2 & 51.9 & 26.2 & 0.0118 \\
\hline
\end{tabular}

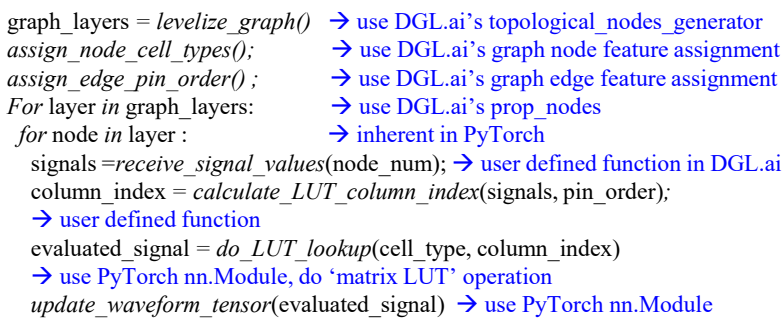

Figure 4: Simulator algorithm and corresponding functionality in PyTorch/DGL.ai

functionality that can be found in the PyTorch and DGL.ai packages. As can be seen, the graph-oriented nature of the DGL.ai package, as well as its integration into PyTorch, makes the coding of these tasks concise and dense - we only need to use readily availables functions from the packages to implement simulation needed tasks. Another convenience is batching across cycles and same logic level gates is inherent in the code.

Experimental Results: Table 1 shows a summary of the benchmarks we ran for our GPU accelerated oblivious, two-value cycle based simulator experiments. Table 2 shows the speedup results. CPU baseline runs were performed on an Intel Xeon CPU E5 in multi-threading mode using a commercial event-based simulation tool. The GPU platform we chose was an NVIDIA Tesla V100 with 16GB memory.

While we are able to attain at least 20X speedup, we observe that the speedup fluctuates somewhat across different benchmarks. We believe this is due to two main reasons. One is that sequential elements that are not simulated take up memory space, so benchmarks with larger portions of their cells being sequential will have reduced amounts of cross cycle parallelism which hurts speedup. Second is that oblivious simulators do not scale well with low activity factors, such as in the RISC-V benchmark.

Interestingly, we may argue that 20X speedup is exaggerated, given the simplistic nature of our scenario's simulator criteria compared to the full-fledged requirements of a commercial simulator tool. However, at the same time we may argue $20 \mathrm{X}$ is conservative considering how un-optimized our simulator is. Nevertheless, we see through this demonstration how higher level API frameworks and packages can help greatly in lowering the barrier of entry into exploring accelerated simulation on GPUs. Along with improvements on the GPU hardware platform (Section 4), the door is wide-open for further research in this area [14].

\begin{tabular}{lll}
\hline Data Center GPU & NVIDIA Tesla V100 & NVIDIA A100 \\
\hline GPU Codename & GV100 & GA100 \\
\hline GPU Architecture & NVIDIA Volta & NVIDIA Ampere \\
\hline SMs & 80 & 108 \\
\hline FP32 Cores / SM & 64 & 64 \\
\hline FP32 Cores / GPU & 5120 & 6912 \\
\hline FP64 Cores / SM & 32 & 32 \\
\hline FP64 Cores / GPU & 2560 & 3456 \\
\hline INT32 Cores / SM & 64 & 64 \\
\hline INT32 Cores / GPU & 5120 & 6912 \\
\hline Tensor Cores / SM & 8 & 4 \\
\hline Tensor Cores / GPU & 640 & 432 \\
\hline Peak FP64 Tensor TFLOPS & NA & 19.5 \\
\hline Peak INT4 Tensor TOPS & NA & $1248 / 2496^{3}$ \\
\hline GPU Boost Clock & $1530 \mathrm{MHz}$ & $1410 \mathrm{MHz}$ \\
\hline Texture Units & 320 & 432 \\
\hline Memory Interface & $4096-\mathrm{bit} \mathrm{HBM2}$ & $5120-\mathrm{bit} \mathrm{HBM2}$ \\
\hline Memory Size & $32 \mathrm{~GB} / 16 \mathrm{~GB}$ & $40 \mathrm{~GB}$ \\
\hline Memory Data Rate & $877.5 \mathrm{MHz}$ DDR & $1215 \mathrm{MHz}$ DDR \\
\hline Memory Bandwidth & $900 \mathrm{~GB} / \mathrm{sec}$ & $1555 \mathrm{~GB} / \mathrm{sec}$ \\
\hline L2 Cache Size & $6144 \mathrm{~KB}$ & $40960 \mathrm{~KB}$ \\
\hline Shared Memory Size / SM & Configurable up to 96 KB & Configurable up to 164 KB \\
\hline Register File Size / SM & $256 \mathrm{~KB}$ & $256 \mathrm{~KB}$ \\
\hline Register File Size / GPU & $20480 \mathrm{~KB}$ & $27648 \mathrm{~KB}$ \\
\hline Figure 5: Compar & & \\
\hline
\end{tabular}

Figure 5: Comparison of Volta and Ampere GPUs. The table is excerpted from [15]. Massive improvements in memory capabilities will help GPU accelerated simultion. 1) Peak rates are based on the GPU boost clock. 2) Four Tensor Cores in an A100 SM have 2x the raw FMA computational power of eight Tensor Cores in a GV100 SM. 3) Effective TOPS/TFLOPS using the new sparsity feature.

\section{Opportunity: Advancements in GPU Hardware and Programming API}

GPUs are increasingly used for general-purpose computing as many applications find the potential for parallel acceleration attractive. This is especially true for machine learning applications that have recently found great success running on GPU hardware platforms. GPU hardware design companies such as NVIDIA are constantly looking to improve upon their platforms, both with new architecture and programming API (for NVIDIA GPUs, the CUDA API) features. Key focuses of many new features center around increasing asynchrony in operation and reducing latencies in communication, memory, and I/O $[1,11]$. These optimization targets overlap with many of the challenges faced in GPU accelerated simulation, such as memory bandwidth and storage capacity requirements $[4,5]$, communication overheads between computation and memory [2-6], and thread occupancy rates in the face of complex scheduling $[2,3,6]$. Thus, next we introduce which new 
features of the newest to be released NVIDIA GPU architecture Ampere [1] might be helpful to further the research area of accelerated GPU simulation.

First, there are many memory oriented optimizations that will directly improve GPU accelerated simulation prospects. Compared to the former generation GPU Volta, Ampere (Figure 5) has massively increased L2 cache size and near double the memory bandwidth, which will help in lowering memory read/write latencies. Asynchronous memory copy [1] can transfer data directly from global memory into shared memory. This memory copy gives a direct performance boost, as it bypasses thread resources (such as registers), and helps double buffered data movement - it gives GPU accelerated simulation the opportunity to load the next set of signal waveforms while evaluating the current set. L2 cache residency control [1] provides an address range up to $128 \mathrm{MB}$, which means larger netlist and signal data can be persistently cached. CUDA virtual memory [1] management can help with programming complex memory management schemes such as those in $[2,3]$.

In addition, fine grained synchronization [1] will help thread occupancy and programming. Researchers will have fewer restrictions when thinking about optimizing thread block occupancy when developing their GPU accelerated simultion algorithms and code. Asynchronous barriers [1] may allow new scheduling algorithms in simulation that keep threads occupied. New Nsight compute [16] provides visual roofline analysis to evaluate kernel characteristics of compute and memory capacities that can aid in informing which portions of simulation need to be further optimized, or which tradeoffs are necessary for overall optimization.

With so many new features available that directly aid many challenges faced, there is ample motivation to further explore the topic of GPU accelerated simulation.

\section{Conclusion: Opportunity and Challenge}

By drawing learnings from prior art, demo-ing a simplistic oblivious simulator that puts forth the idea that ML frameworks and packages easily lower the startup research cost for the topic, and observing how new GPU platform features provide new avenues than tie-in to enhancing GPU accelerated simulation prospects, we conclude that GPU accelerated simulation is an exciting research area rife with opportunities. Of note is that though the demonstration in Section 3 restricts the scope to exploiting massive stimuli and instance parallelism for gate level simulation of logic gates, this type of thinking can be expanded to different levels of simulation. RTL simulation may benefit from stimuli parallelism similarly, perhaps in a system where thousands of the same DUT are instantiated, each running a different stimulus in parallel. It is possible such a method could greatly improve GPU utilization, leading to large speedups and working around Amdahl's law bottlenecks (such as serial code in testbenches) that would be encountered when attempting to 'strong scale' single testbenches.

The improvements to the GPU platform are also not specific to gate level simulation. They provide expansive opportunities for improvement in GPU accelerated simulation. New algorithms and methods may emerge to tackle further issues encountered in comprehensive accelerated simulation, such as supporting arbitrary code in RTL simulation, inclusion of fast simulation of sequential components, and adding support for verification oriented constructs and different timing modalities (zero delay cycle mode, glitch filtering modes, etc).

\section{References}

[1] NVIDIA A100 Tensor Core GPU Architecture.

https://www.nvidia.com/content/dam/en-zz/Solutions/Data-Center/nvidiaampere-architecture-whitepaper.pdf, 2020

[2] Stefan Holst, Michael E. Imhof, and Hans-Joachim Wunderlich. High-throughput logic timing simulation on gpgpus. ACM Trans. Des. Autom. Electron. Syst., 20(3), June 2015.

[3] Yuhao Zhu, Bo Wang, and Yangdong Deng. Massively parallel logic simulation with gpus. ACM Trans. Des. Autom. Electron. Syst., 16(3), June 2011.

[4] A. Sen, B. Aksanli, M. Bozkurt, and M. Mert. Parallel cycle based logic simulation using graphics processing units. In 2010 Ninth International Symposium on Parallel and Distributed Computing, pages 71-78, 2010.

[5] Debapriya Chatterjee, Andrew Deorio, and Valeria Bertacco. Gate-level simulation with gpu computing. ACM Trans. Des. Autom. Electron. Syst., 16(3), June 2011.

[6] H. Qian and Y. Deng. Accelerating rtl simulation with gpus. In 2011 IEEE/ACM International Conference on Computer-Aided Design (ICCAD), pages 687-693, 2011.

[7] S. Vinco, V. Bertacco, D. Chatterjee, and F. Fummi. Saga: Systemc acceleration on gpu architectures. In DAC Design Automation Conference 2012, pages 115-120, 2012 .

[8] B. Catanzaro, K. Keutzer, and Bor-Yiing Su. Parallelizing cad: A timely research agenda for eda. In 2008 45th ACM/IEEE Design Automation Conference, pages 12-17, 2008.

[9] PyTorch. https://pytorch.org, 2020.

[10] DGL.ai. https://www.dgl.ai, 2020

11] CUDA Toolkit 11.0. https://developer.nvidia.com/cuda-downloads, 2020.

[12] Yibo Lin, Shounak Dhar, Wuxi Li, Haoxing Ren, Brucek Khailany, and David Z. Pan. Dreamplace: Deep learning toolkit-enabled gpu acceleration for modern vlsi placement. In Proceedings of the 56th Annual Design Automation Conference 2019, DAC '19, New York, NY, USA, 2019. Association for Computing Machinery.

[13] Thomas N. Kipf and Max Welling. Semi-supervised classification with graph convolutional networks, 2016.

[14] Yanqing Zhang, Haoxing Ren, Ben Keller, and Brucek Khailany. Problem c: Gpu accelerated logic re-simulation. In 2020 IEEE/ACM International Conference on Computer-Aided Design (ICCAD), 2020.

[15] NVIDIA Ampere Architecture In-Depth. https://developer.nvidia.com/blog/nvidiaampere-architecture-in-depth, 2020.

[16] NVIDIA Nsight Compute. https://developer.nvidia.com/nsight-compute, 2020. 